\title{
Fractal Dimension of Magnetic Domain Walls in CoFe/Pt Multilayers
}

\author{
Kang-Soo Lee ${ }^{1}$, Dong-Hyun Kim ${ }^{2}$, and Sug-Bong Choe ${ }^{1 *}$ \\ ${ }^{1}$ Department of Physics, Seoul National University, Seoul 151-742, Korea \\ ${ }^{2}$ Department of Physics, Chungbuk National University, Cheongju 361-763, Korea
}

(Received 3 May 2010, Received in final form 1 July 2010, Accepted 5 July 2010)

\begin{abstract}
We present the fractal properties of the magnetic domain walls in $\left(5-\AA \mathrm{Co}_{90} \mathrm{Fe}_{10} / 10-\AA \mathrm{Pt}\right)_{n}$ multilayer films with perpendicular magnetic anisotropy for the number of repeats $n$ ( 1 to 5 ). In these films, the magnetization reversed due to the domain wall propagation throughout the films with rare nucleations. As $n$ increased, it was observed that the jaggedness of the domain walls increased noticeably, which is possibly due to the accumulation of irregularities at the layer interfaces. The jaggedness of the domain walls was analyzed in terms of the fractal dimension by use of the ruler method, and it was revealed that the fractal dimension significantly changed from $1.0 \pm 0.002$ to $1.3 \pm 0.05$ as $n$ increased from 1 to 5 .
\end{abstract}

Keywords : fractal dimension, magnetic thin film, magnetization reversal

\section{Introduction}

Uniformity and controllability of the magnetic properties in ferromagnetic films and nanostructures are paramount in achieving reliability in magnetic device applications such as the magnetic domain-wall racetrack memory [1], logic devices [2], and various upcoming technologies [3]. The uniformity of the films can be examined by observing the magnetization reversal processes which display the magnetic properties clearly. Magnetization reversal in ferromagnetic films with perpendicular magnetic anisotropy occurs by several distinct mechanisms - the wall-motion from existing domains and the nucleation of domains at random positions - depending on the film structure and composition as well as the fabrication conditions [4-7]. To understand these reversal processes with magnetic properties, many theoretical models, such as the random-field Ising model, the random-bond Ising model [8], the thermally activated relaxation model [911], and so on, have been proposed and tested. In these models, randomness plays a crucial role. The degree of randomness governs the domain dynamics and, consequently, causes distinct domain wall structures. From a theoretical point of view, the magnetization reversal occurs through randomly distributed defects in amorphous or

*Corresponding author: Tel: +82-2-880-9254

Fax: +82-2-884-9254, e-mail: sugbong@snu.ac.kr poly-crystallized materials and, consequently, such nonlinear stochastic effects result in the fractal nature of the magnetic domain patterns [12]. Many studies have examined the fractal properties of the magnetic domains [914] and domain walls $[9,12]$. The fractal dimension provides a unique characterization parameter for domain interface formation in accordance with non-uniformity. Here, we investigate the fractal properties of $\mathrm{CoFe} / \mathrm{Pt}$ multilayered films to quantitatively analyze the effect of the number of layers on the domain wall jaggedness.

\section{Experiments}

50- $\AA \mathrm{Ta} / 25-\AA \mathrm{Pt} /\left(5-\AA \mathrm{Co}_{90} \mathrm{Fe}_{10} / 10-\AA \mathrm{Pt}\right)_{n}$ films were deposited on a $\mathrm{Si}$ substrate with a natural $\mathrm{SiO}_{2}$ layer via dc-magnetron sputtering and the films were produced with various numbers of repeats $n$ (from 1 to 5 ). Here we chose to use $\mathrm{CoFe}$ films rather than Co films because CoFe films exhibit an almost amorphous phase with small polycrystalline grains. From the magneto-optical Kerr effect measurement, all the films were revealed to exhibit squared out-of-plane hysteresis loops as shown in Fig. 1, evidencing the strong perpendicular magnetic anisotropy. The coercive fields were revealed to increase monotonically from 113 Oe to 363 Oe as $n$ increased from 1 to 5 .

The films were first saturated under a sufficiently large magnetic field (3,300 Oe) and then, by applying short pulses of reversed magnetic fields (similar strength with 


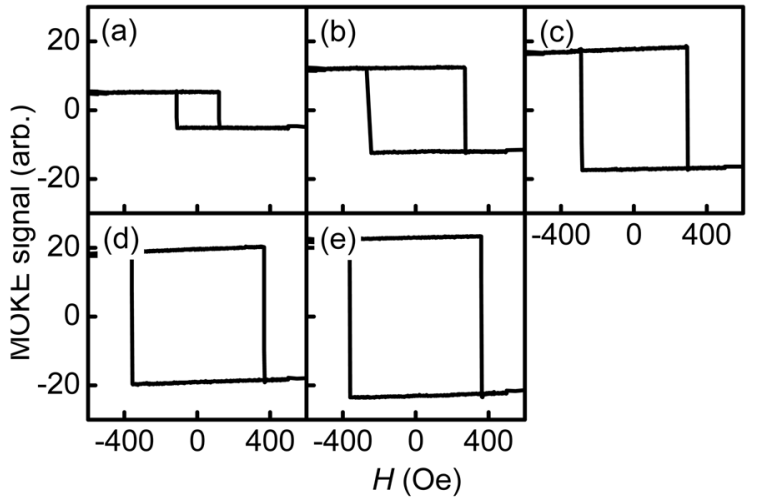

Fig. 1. Out-of-plane magnetic hysteresis loops of the films with different repetition numbers $n$ of the CoFe/Pt layers, i.e. $n=$ (a) 1, (b) 2, (c) 3, (d) 4, and (e) 5, respectively. The sweeping rate of the magnetic field is $66 \mathrm{Oe} / \mathrm{sec}$ within the range of $\pm 2,400$ Oe. The coercive field of each film was measured to be (a) 113 Oe, (b) 272 Oe, (c) 295 Oe, (d) 360 Oe, and (e) 363 Oe, respectively.

the coercive field, 113 Oe, 272 Oe, 295 Oe, and 360 Oe for the samples with $n=1,2,3,4$, and 5 , respectively), a few domain nuclei were formed at quenched positions (localized soft magnetic defects). Rather weak magnetic fields, rather than coercive fields, triggered the domain wall to propagate slowly $(10-20 \mu \mathrm{m} / \mathrm{s})$ from the nuclei. The typical domain images are illustrated in Fig. 2(a). The gray contrast corresponds to the domain images at different times after applying the magnetic field. Since the domain wall jaggedness is known to depend on the wall propagation speed [11, 15], we applied different magnetic fields (42 Oe, $65 \mathrm{Oe}, 197 \mathrm{Oe}, 280 \mathrm{Oe}$, and 291 Oe for the samples with $n=1,2,3,4$, and 5 , respectively) to induce a similar domain-wall velocity of about $15 \mu \mathrm{m} / \mathrm{s}$, where the randomness of the films becomes dominant in determining the jaggedness of the domain walls. After the saturation of the jaggedness growth via sufficient domain wall propagation reached about a few millimeters, the domain patterns were captured by a time-resolved magneto-optical Kerr effect microscope, which was capable of up to $\times 1,000$ magnification with an objective lens with a numerical aperture of 0.75 with a spatial resolution of $0.45 \mu \mathrm{m}$. The captured domain wall images were then analyzed after some image processing: the subtraction from pre-captured background images, the black-andwhite conversion, and then the digitization of the domain wall positions. The typical domain wall structures are shown in Fig. 2(b). The domain wall profiles were then finally obtained as an array of the domain wall positions. All the image analyses were carried out under the same prescribed conditions.
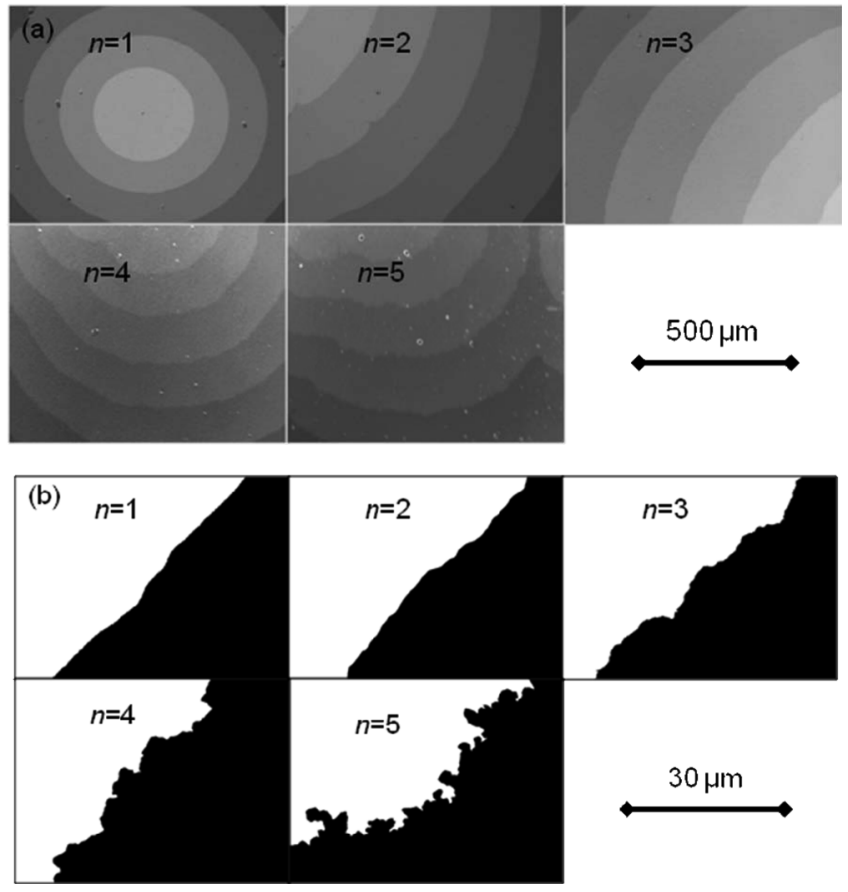

Fig. 2. (a) Typical magnetic domain expansion from nucleation sites, which are sometimes at the edge (or middle) of the film, with low magnification $(\times 25)$. The gray contrasts correspond to the domain images taken at different times after applying the magnetic field. (b) Typical magnetic domain images with high magnification $(\times 1,000)$ after image processing, which comprises background subtraction and black-andwhite conversion.

The fractal properties of the domain wall profile were then analyzed by the ruler method. It is well known that the fractal dimension $D_{\mathrm{F}}$ can be estimated from the scaling relation among the geometrical parameters, such as the perimeter length measured by various rulers with different lengths and the area size measured by different square cells [16]. Here, we determined the fractal dimensions of the domain walls by measuring length $L$ of the domain interfaces on the image by changing the ruler size $s$. The length $L$ of the domain wall in a captured image changed depending on the ruler size $s$. In log-log plots of the domain wall length $L$ with respect to the ruler size $s$ as shown in Fig. 3, the slopes determine the fractal dimensions according to $D_{\mathrm{F}}=1-$ slope. The steeper the slope is, the larger the fractal dimension is.

\section{Results and Discussion}

All the films exhibit clear circular expansion of the magnetic domains from the rare nuclei at some localized positions as seen with the low magnification images in Fig. 2(a), which show that the films are fairly homogene- 


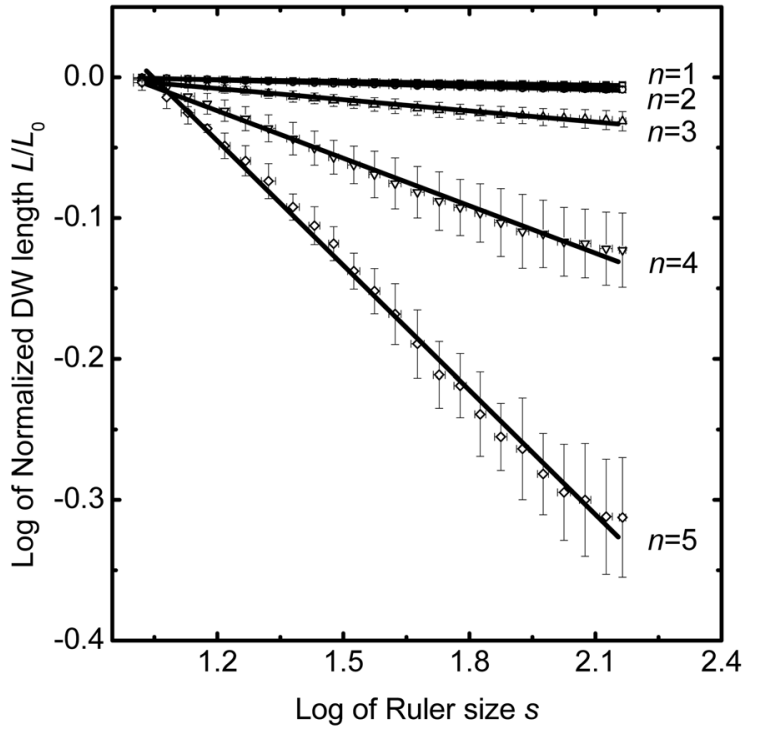

Fig. 3. Log-log plots of the domain wall length with respect to the ruler size for each film. The symbols with error bars indicate the statistics from several tens of images for each film. The lines show the best linear fits.

ous and isotropic from a macroscopic point of view. However, zooming onto the microscopic details, the domain wall profiles turned out to be significantly different from each other as shown in the high magnification images in Fig. 2(b). Each domain wall profile corresponds to the distinct fractal properties which are related to microscopic homogeneity.

Fig. 3 shows the log-log plots of the domain wall structures, where the abscissa is the ruler size $s$ and the ordinate is the normalized domain wall length $L / L_{0}$ for the domain wall profiles shown in Fig. 2(b). Here $L_{0}$ is the domain wall length of each image measured by the smallest ruler which was determined by the spatial resolution of the microscope. Then we collected statistics of the normalized domain wall lengths $L / L_{0}$ according to the ruler length $s$. The clear linear dependence supports the validity of our approach with the fractal analysis and the actual fractality of the magnetic domain walls on our films. From the linear dependence, we could deduce the fractal dimension $D_{\mathrm{F}}$ of the domain walls on each film from the relation $L \propto s^{1-D F}$ [14]. Interestingly, the fractal dimension was revealed to drastically increase with increases in $n$, as shown in Fig. 4. The fractal dimension significantly changed from $1.0 \pm 0.002(n=1)$ to $1.3 \pm 0.05$ $(n=5)$. This change might be ascribed to the accumulation of irregularities formed at the interfaces between the $\mathrm{CoFe}$ and Pt layers. The irregularities then caused magnetic pinning during the domain wall propagation and, consequently, induced the domain wall to be roughened.

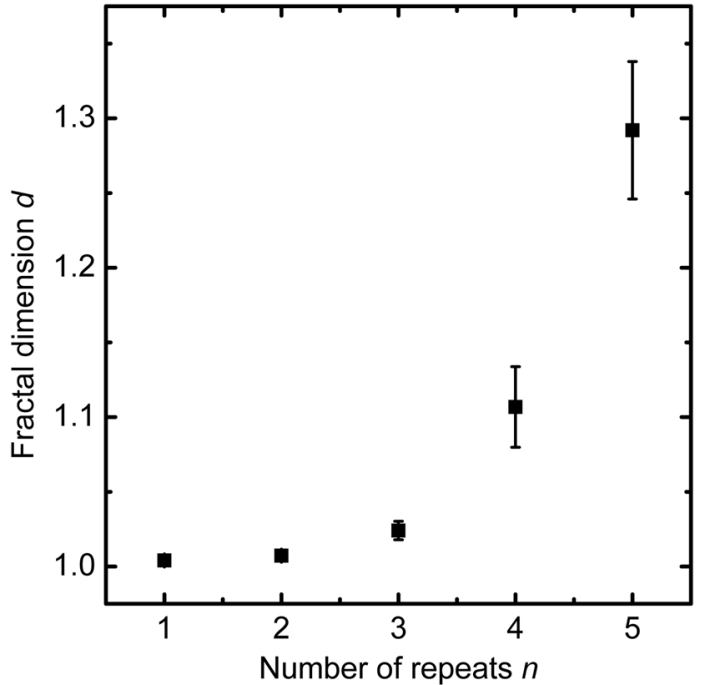

Fig. 4. Fractal dimensions with respect to the number of repeats.

The fractal properties of magnetic domains have been well described in the thermo-activated relaxation model of domain wall propagation $[9,11,14]$. In this model, the domain evolution occurs via the Barkhausen jumps of the domain walls by overcoming the breakaway energy $R$ with the help of thermal activation. According to the Arrhenius law, the probability $p$ for the thermally-activated depinning is given by $p \sim \exp (-R / T)$ for temperature $T$. In Ref. 8 , the thermal activation process is described by a single characteristic parameter $z$ as defined by

$$
z=\frac{t}{\xi} \frac{V_{A} M_{s}^{2}}{T}
$$

where $t$ and $\xi$ are the film thickness and the lattice cell size, respectively. Here, $V_{\mathrm{A}}$ is the activation volume and $M_{\mathrm{S}}$ is the saturation magnetization. Consequently, their results show that the fractal dimension $D_{\mathrm{F}}$ monotonically increased as z increased (For details, see Fig. 6 in Ref. 8). For our case, the total thickness $t$ increased linearly with the number of repeats, whereas $\xi$-the characteristic length of microscopic magnetic uniformity - decreased due to the accumulation of the interfacial irregularities. Since the activation volume $V_{\mathrm{A}}$ and the saturation magnetization $M_{\mathrm{S}}$ are almost unchanged for all the samples, it is expected that $z$ increased with respect to the number of repeats, resulting in monotonic increments of the fractal dimension.

\section{Conclusion}

The fractal properties of the magnetic domain walls were experimentally investigated in ferromagnetic $\mathrm{CoFe} /$ 
Pt multilayered films with perpendicular magnetic anisotropy. The jaggedness of the magnetic domain walls increased and, consequently, the fractal dimension also increased with the increasing repetition number of the $\mathrm{CoFe} / \mathrm{Pt}$ layers. This is possibly ascribed to the accumulation of interfacial defects due to increasing the number of repeats.

\section{Acknowledgement}

This work was supported by NRF funded by MEST through Mid-career Researcher Program (2007-0056952, 2009-0084542).

\section{References}

[1] S. S. P. Parkin, M. Hayashi, and L. Thomas, Science 320, 190 (2008).

[2] D. A. Allwood, G. Xiong, C. C. Faulkner, D. Atkinson, D. Petit, and R. P. Cowburn, Science 309, 1688 (2005).

[3] G. A. Prinz, Science 282, 1660 (1998).

[4] J. Pommier, P. Meyer, G. Pénissard, and J. Ferré, Phys.
Rev. Lett. 65, 2054 (1990).

[5] S.-B. Choe and S.-C. Shin, Appl. Phys. Lett. 80, 1791 (2002).

[6] S.-B. Choe and S.-C. Shin, J. Appl. Phys. 87, 5076 (2000).

[7] J. Y. Ahn, N. J. Lee, and T. H. Kim, J. Magnetics 14, 144 (2009).

[8] H. Ji and M. O. Robbins, Phys. Rev. A. 44, 2538 (1991).

[9] G. V. Sayko, A. K. Zvezdin, T. G. Pokhil, B. S. Vvedensky, and E. N. Nikolaev, IEEE Trans. Magn. 28, 2931 (1992).

[10] D.-H. Kim, Y.-C. Cho, S.-B. Choe, and S.-C. Shin, Phys. Stat. Sol. 241, 1669 (2004).

[11] A. Kirilyuk, J. Ferré, and D. Renard, Europhys. Lett. 24, 403 (1993).

[12] B.-S. Han, D. Li, D.-J. Zheng, and Y. Zhou, Phys. Rev. B 66, 014433 (2002).

[13] B. E. Bernacki and M. Mansuripur, J. Appl. Phys. 69, 4960 (1991).

[14] D.-H. Kim, Y.-C. Cho, S.-B. Choe, and S.-C. Shin, Appl. Phys. Lett. 82, 3698 (2003).

[15] A. Kirilyuk, J. Ferré, V. Grolier, J. P. Jamet, and D. Renard, J. Magn. Magn. Mater. 171, 45 (1996).

[16] J. Feder, Fractals, Plenum, New York (1988). 